
score; survival; patient selection

\title{
Development of a prognostic scoring system for patients with advanced cancer enrolled in immune checkpoint inhibitor phase 1 clinical trials
}

Shiraj Sen ${ }^{1,2}$, Kenneth Hess ${ }^{3}$, David S Hong ${ }^{2}$, Aung Naing ${ }^{2}$, Sarina Piha-Paul ${ }^{2}$, Filip Janku ${ }^{2}$, Siqing Fu ${ }^{2}$, Ishwaria M Subbiah ${ }^{1}$, Holly Liu ${ }^{2}$, Rahil Khanji ${ }^{2}$, Le Huang ${ }^{2}$, Shhyam Moorthy ${ }^{2}$, Daniel D Karp ${ }^{2}$, Apostolia Tsimberidou², Funda Meric-Bernstam ${ }^{2}$ and Vivek Subbiah ${ }^{\star 2}$

${ }^{1}$ Division of Cancer Medicine, The University of Texas MD Anderson Cancer Center, Houston, TX 77030, USA; ${ }^{2}$ Department of Investigational Cancer Therapeutics (Phase I Clinical Trials Program), The University of Texas MD Anderson Cancer Center, Houston, TX 77030, USA and ${ }^{3}$ Department of Biostatistics, The University of Texas MD Anderson Cancer Center, Houston, TX 77030, USA

Background: We sought to develop a prognostic scoring system to aid in patient selection for immune checkpoint inhibitor (ICl) phase 1 clinical trials.

Methods: Clinical data from patients treated in phase $1 \mathrm{ICl}$ clinical trials at MD Anderson (MDA) Center were analysed. Seventeen clinical factors were studied. Recursive partitioning analysis, a tree-based model, was used to develop a regression tree and identify optimal cut-points based on differences in survival for each clinical factor. A Cox proportional hazards regression model was then used to identify factors independently affecting overall survival. A prognostic scoring system was subsequently developed.

Results: A total of 172 patients (105 CTLA4- and 67 PD1-based) were analysed. Seven factors were independently associated with worse overall survival (OS): age $>52$ years (hazard ratio (HR) 1.59, 95\% confidence interval (CI) 1.1-2.4), Eastern Cooperative Oncology Group performance status $>1$ ( $\mathrm{HR} 2.81,95 \% \mathrm{Cl} 1.3-6.3)$, lactate dehydrogenase $>466$ (which is $0.75 \times$ the upper limit of normal at our institution) (HR 2.1,95\% Cl 1.4-3.2), platelet count $>300 \times 10^{3} \mu \mathrm{L}^{-1}$ (HR 1.8, 95\% Cl 1.2-2.8), absolute neutrophil count $>4.9 \times 10^{3} \mu \mathrm{L}^{-1}$ (HR 2.3, 95\% Cl 1.5-3.5), absolute lymphocyte count $<1.8 \times 10^{3} \mu \mathrm{L}^{-1}$ (HR 3.3, 95\% Cl 1.9-5.7), and liver metastases (HR 1.8, 95\% Cl 1.2-2.6). An index was created by dividing the cohort into risk groups based on the number of factors present: $0-2,3,4$, or 5-6. Median OS was 24.2 months, 11.6 months, 8.0 months, and 3.8 months for patients with $0-2,3$, 4, or 5-6 risk factors, respectively; log-rank test, $P<0.0001$. The Harrell $c$-index of this scoring system was 0.72 , indicating better predictability than the Royal Marsden Hospital score (c-index 0.67) and MDA score (c-index 0.61).

Conclusions: We have developed a novel 'MDA-ICl' prognostic scoring system for patients treated in phase $1 \mathrm{ICl}$ clinical trials. Prospective evaluation and external validation is warranted and may help aid patient selection for future clinical trials.

When patients with advanced cancers exhaust all US FDAapproved cancer therapies, oncologists must decide whether to recommend hospice care or seek eligibility for phase 1 trials.
Although phase 1 trials have been proven to be generally safe (Arkenau et al, 2008), it remains difficult to estimate prognosis in patients with advanced cancer (Bachelot et al, 2000). This makes it

*Correspondence: Dr V Subbiah; E-mail: vsubbiah@mdanderson.org

Received 4 July 2017; revised 5 December 2017; accepted 6 December 2017; published online 20 February 2018

(C) 2018 Cancer Research UK. All rights reserved 0007 - 0920/18 
challenging to select the appropriate patients to enroll onto phase 1 trials. In an effort to aid oncologists in prognosticating patients for phase 1 clinical trials, the Royal Marsden Hospital (RMH)(Arkenau et al, 2008), and MD Anderson (MDA) (Wheler et al, 2012) prognostic scoring systems were developed. The RMH score incorporates serum albumin concentration, serum lactate dehydrogenase concentration, and number of metastatic disease sites. The MDA scoring system adds negative prognostic value to Eastern Cooperative Oncology Group (ECOG) performance status (PS) $\geqslant 1$ and GI cancers to improve upon the RMH score. Both scoring systems were developed and validated in the era of chemotherapy and targeted therapy.

In recent years, immune checkpoint inhibitor (ICI)-based phase 1 clinical trials have emerged at the forefront of oncology drug development. Although durable responses have been noted in metastatic melanoma and multiple solid tumours including nonsmall cell lung cancer, renal cell cancer, and head and neck cancer (Borghaei et al, 2015; Larkin et al, 2015), overall response rates remain low. In contrast to chemotherapy and targeted therapy, ICI-based therapy is not directly cytotoxic, has unique mechanisms of action by modulating the tumour microenvironment, and is accompanied by a very different side effect profile (Michot et al, 2016). We do not know if the RMH and MDA scores may be applicable to ICI trials and there is a need to develop a prognostic scoring system for ICI-based phase 1 clinical trials.

The objective of this study was to evaluate patients enrolled on phase 1 ICI trials in our clinic at the MD Anderson Cancer Center (Houston, TX, USA) in order to identify risk factors independently affecting overall survival (OS) and develop a prognostic scoring system for phase 1 ICI trials. We have identified seven clinical factors that can independently predict worse OS: age $>52$ years, ECOG PS $>1, \mathrm{LDH}>0.75 \times \mathrm{ULN}$, platelet count $>300 \times 10^{3}$ $\mu \mathrm{L}^{-1}$, absolute neutrophil count $>4.9 \times 10^{3} \mu \mathrm{L}^{-1}$, absolute lymphocyte count $<1.8 \times 10^{3} \mu \mathrm{L}^{-1}$, and the presence of liver metastases.

\section{PATIENTS AND METHODS}

We reviewed the electronic medical records of 172 patients with advanced cancer enrolled in phase 1 ICI-based clinical trials (antiCTLA4 and anti-PD1 trials) at the MD Anderson Clinical Department of Investigational Cancer Therapeutics (Phase 1 Clinical Trials Program) from January 2013 to November 2015. Investigational regimens available for patient enrollment were determined by protocol availability at the time each patient presented to clinic. Each ICI regimen administered and number of patients on each trial is listed in Supplementary Table 1. No patients with preexisting autoimmune diseases requiring diseasemodifying therapy were included in this study.

Ethics statement. This analysis as well as treatment on each clinical trial was done with approval of the MD Anderson Cancer Center Institutional Review Board. Participants gave informed written consent.

Endpoints and statistical methods. The purpose of this study was to identify risk factors associated with OS in patients with advanced cancers treated in a phase I clinic with ICI-based therapy. The primary endpoint in evaluating potential risk factors was OS, which was measured from cycle 1 day 1 of treatment with an ICI-based therapy until death from any cause or last follow-up. Patients still alive at the time of analysis were censored for survival at the time of their last follow-up. Descriptive statistics were used to detail baseline patients' characteristics. Categorical data were detailed with contingency tables including counts and percentages. Continuous measures were summarised with descriptive statistical measures, as well (i.e., median (range)). Survival curves were estimated by the Kaplan-Meier method.

The following covariates have previously demonstrated prognostic significance in the literature and were analysed in univariate analysis: age ( $>52 v s \leqslant 52$ years), gender, number of metastases ( $>2 v s \leqslant 2$ sites of metastases), serum haemoglobin level $(>10.1$ $\left.v s \leqslant 10.1 \mathrm{~g} \mathrm{dl}^{-1}\right)$, absolute lymphocyte count $\left(>1.8 v s \leqslant 1.8 \times 10^{3}\right.$ cells $/ \mu \mathrm{L}$ ), presence of lung, liver, bone, or brain metastases (yes $v s$ no), ECOG PS $(>1 v s \leqslant 1)$, albumin $\left(>3.5 v s \leqslant 3.5 \mathrm{~g} \mathrm{dl}^{-1}\right)$, lactate dehydrogenase $\left(>466 \mathrm{vs} \leqslant 466 \mathrm{IU}^{-1}\right)$, total white blood cell count $\left(>6.1 v s \leqslant 6.1 \times 10^{3} \mu \mathrm{L}^{-1}\right)$, platelet count $(>300 v s$ $\left.\leqslant 300 \times 10^{3} \quad \mu \mathrm{L}^{-1}\right), \quad$ absolute neutrophil count $(>4.9$ vs $\left.\leqslant 4.9 \times 10^{3} \mu \mathrm{L}^{-1}\right)$, neutrophil-to-lymphocyte ratio $(>4 v s \leqslant 4$ ), and platelet-to-lymphocyte ratio $(>498$ vs $\leqslant 498)$. Recursive partitioning analysis, using a regression tree-based model, was performed for each numeric variable listed above to find the optimal cut-point.(Marshall, 2001)

The RMH score and MDACC score was also calculated for each patient, based on the clinical and laboratory values obtained the day the patient started treated on immune checkpoint-inhibitorbased therapy in the phase 1 clinic. The RMH score includes the following poor prognostic variables: albumin $<3.5 \mathrm{~g} \mathrm{dl}^{-1}$, $\mathrm{LDH}>\mathrm{ULH}\left(618 \mathrm{IU}^{-1}\right.$ at our institution), and two or greater sites of metastatic disease (Arkenau et al, 2008). The MD Anderson score also includes ECOG PS and GI primary tumours as negative prognostic variables.(Wheler et al, 2012)

Cox proportional hazards regression model was used to conduct multivariate analysis to identify factors independently affecting OS. A prognostic scoring system was then established whereby each negative prognostic factor was assigned a point. Survival by prognostic score was estimated by the Kaplan-Meier method and compared to survival estimates using the RMH and MDACC prognostic scoring system in our patient population.

We examined the predictive ability of prognostic factors for survival with the Harrell c-statistic (Harrell, Lee and Mark, 1996); a higher c-statistic indicates greater predictive ability. All statistical tests were two-sided and $P<0.05$ was considered statistically significant. Statistical analyses were conducted with TIBCO Spotfire S-Plus version 8.2 for Windows.

\section{RESULTS}

Of the 172 patients treated with ICI therapy (105 CTLA4- and 67 PD1-based) in the Department of Investigational Cancer Therapeutics (Phase 1 clinic), the median age of all patients was 60 years (range: $19-86$ years) and 87 (51\%) were males. The most common tumour types treated included renal cell carcinoma $(n=25 ; 14.5 \%)$, non-small cell lung cancer $(n=21 ; 12.2 \%)$, melanoma $(n=16 ; 9.4 \%)$, sarcoma $(n=16 ; 9.4 \%)$, gastrointestinal stromal tumours $(n=10 ; 5.8 \%)$, thyroid cancer $(n=7 ; 4.1 \%)$, prostate cancer $(n=6 ; 3.4 \%)$, and colorectal cancer $(n=6 ; 3.4 \%)$, anal cancer $(n=5 ; 2.9 \%)$ (Figure $1 \mathrm{~A})$. Fifty-four patients $(31 \%)$ had three or more sites of metastatic disease, including 100 patients (56\%) with lung metastases, 65 patients (38\%) with liver metastases, $48(28 \%)$ with bone metastases, and $14(8 \%)$ with brain metastases. Other baseline demographics include 57 patients $(33 \%)$ with an elevated baseline $\mathrm{LDH}\left(\geqslant 618 \mathrm{Ul}^{-1}\right.$ at our institution), 20 (12\%) with an elevated baseline absolute neutrophil count $\left(\geqslant 5.4 \times 10^{3} \mu 1^{-1}\right.$ at our institution), and $96(56 \%)$ with decreased baseline absolute lymphocyte count $\left(<1.2 \times 10^{3} \mu \mathrm{l}^{-1}\right.$ at our institution) (Table 1).

Treatment. All patients in this analysis were treated with ICIbased therapies. Sixty-seven patients $(38.9 \%)$ were treated with a single agent ICI, 46 patients (26.7\%) were treated with a combination of an ICI and radiation therapy, 32 patients (18.6\%) 

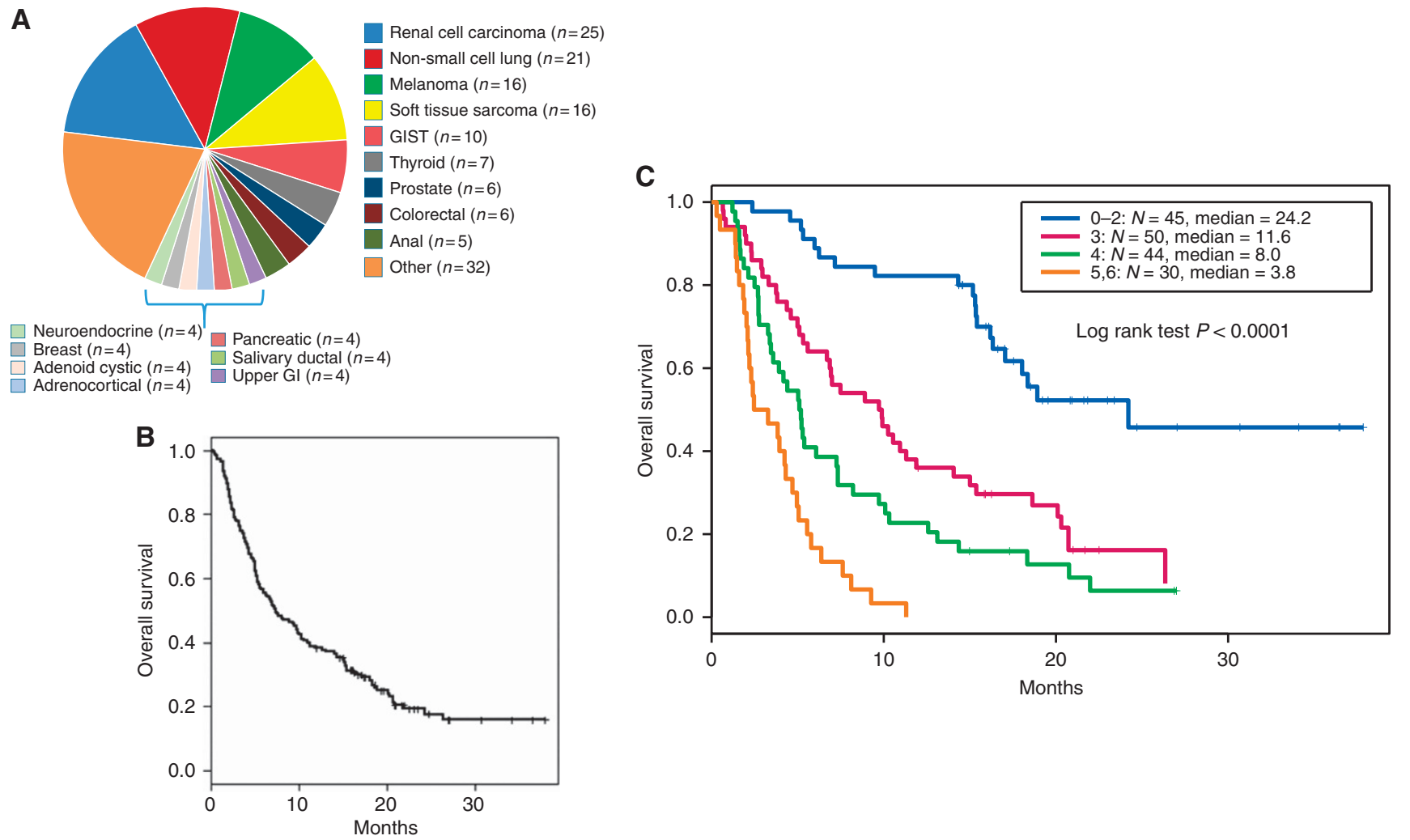

Figure 1. Overall survival in patients treated on phase $1 \mathrm{ICl}$ therapy. (A) Pie chart listing all patients enrolled on immune checkpoint inhibitor phase 1 trials during the time of analysis by tumour type. (B) Kaplan-Meier survival analysis for overall survival in all patients treated on ICl phase 1 trials. (C) Overall survival in patients treated on $\mathrm{ICl}$ phase 1 trials by $\mathrm{MDA} \mathrm{ICl}$ risk score. The cohort is divided into four risk groups based on the number of factors present: 0-2 (blue), 3 (purple), 4 (green), 5-6 (orange). ICl=immune checkpoint inhibitor therapy; MDA=MD Anderson.

were treated with a combination of an ICI and targeted therapy, and 27 patients $(15.7 \%)$ were treated with a combination of an ICI and second immunomodulatory agent.

Survival. Of the 172 patients, there were 134 deaths after a median follow-up of 23.5 months. The median OS for the entire cohort was 7.3 months (95\% CI 5.8-10.3 months) (Figure 1B). Survival rates at 6,12 , and 18 months were 56\% (95\% CI 49-64\%), 38\% (95\% CI $32-46 \%$ ), and 19\% (95\% CI 14-27\%), respectively.

At the time of analysis, five patients (2.9\%) remained on protocol without evidence of disease progression. Of the 167 patients that had been taken off of protocol, 138 (80.2\%) patients were taken off protocol owing to disease progression, $14(8.1 \%)$ patients withdrew consent, $8(4.6 \%)$ patients were taken off protocol owing to death, $6(3.4 \%)$ patients were taken off protocol due to toxicity, and 1 patient was at physician discretion.

Univariate analysis for survival. The factors that were associated with shorter survival in univariate analysis were ECOG performance status $>1$ (hazard ratio (HR) 2.6, 95\% confidence interval (CI) $1.2-5.6, P=0.016$ ), lactate dehydrogenase $>466$ (which is $0.75 \times$ the upper limit of normal at our institution) (HR 2.6, 95\% CI 1.7-3.8, $P=<0.0001$, total white blood cell count $>6.1 \times 10^{3}$ $\mu \mathrm{L}^{-1}$ (HR 1.8, 95\% CI 1.3-2.6, $P=0.0007$ ), platelet count $>300 \times 10^{3} \mu \mathrm{L}^{-1}$ (HR 1.9, 95\% CI 1.3-2.8, $P=0.0005$ ), absolute neutrophil count $>4.9 \times 10^{3} \mu \mathrm{L}^{-1}$ (HR 2.2, 95\% CI 1.5-3.0, $P=<0.0001$ ), neutrophil-to-lymphocyte ratio $>4$ (HR 2.1, 95\% CI 1.4-2.9, $P=<0.0001$ ), presence of liver metastases (HR 1.8, 95\% CI 1.2-2.5, $P=0.0013)$, and platelet-to-lymphocyte ratio $>498$ (HR 3.0, 95\% CI 1.8-5.0, $P<0.0001$ ). Table 2.

Development of a novel prognostic scoring system. Multivariate analysis using Cox proportional hazard regression with manual backward variable selection was used to identify a parsimonious set of factors that independently effect OS. Seven factors were identified as being associated with significantly worse OS: age $>52$ years $(\mathrm{HR} 1.59,95 \%$ CI 1.1-2.4, $P=0.029)$, ECOG performance status $>1$ (HR 2.81, 95\% CI 1.3-6.3, $P=0.011)$, $\mathrm{LDH}>466$ (which is $0.75 \times$ the upper limit of normal at our institution) (HR 2.1, 95\% CI 1.4-3.2, $P=0.0003$ ), platelet count $>300 \times 10^{3} \mu \mathrm{L}^{-1}(\mathrm{HR} 1.8,95 \%$ CI $1.2-2.8, P=0.0064)$, ANC $>4.9 \times 10^{3} \mu \mathrm{L}^{-1}(\mathrm{HR} 2.3,95 \%$ CI $1.5-3.5, P=0.0005)$, ALC $<1.8 \times 10^{3} \mu \mathrm{L}^{-1}$ (HR 3.3, 95\% CI 1.9-5.7, $P=0.0004$ ), and liver metastases (HR 1.8, 95\% CI 1.2-2.6, $P=0.002$ ) (Table 3). A score was then developed whereby one point was assigned for each negative prognostic factor to each patient (Table 4). Only one patient had 0 risk factors, 10 patients had 1 risk factors, and 6 patients had 6 risk factors in our cohort. The cohort was divided into four risk groups based on the number of factors present: $0-2$, 3 , 4, or 5-6. Median OS was 24.2 months (0-2), 11.6 months (3), 8.0 months (4), and 3.8 months (5-6); log-rank test, $P<0.0001$ (Figure 1C). The Harrell c-index of this scoring system was 0.72 (0.08), indicating significant predictability.

Comparison of MDA ICI score with RMH and MDA scores. We then compared the ability of our novel score to prognosticate patients treated on ICI phase 1 trials with the previously used $\mathrm{RMH}$ and MDACC scoring systems. When the RMH score was included in the Cox model, median OS for patients in our cohort of patients treated with checkpoint inhibitor-based therapy was 13.6 months (RMH score $0, n=80$ ), 5.7 months (RMH score $1, n=60$ ), and 4.3 months (RMH score $2-3, n=30$ ), log-rank test $P<0.0001$ (Figure 2A). The c-index for predictability in our cohort was 0.67 (0.06). When the MDACC score (which also incorporates ECOG $\mathrm{PS} \geqslant 1$ and GI tumours as having negative prognostic value) was applied to our cohort, median OS for patients was 13.6 months (MDACC score $0-1, n=76$ ), 7.3 months (MDACC score 2, 
Table 1. Baseline demographics

\begin{tabular}{|c|c|}
\hline Parameter & Number (\%) \\
\hline \multicolumn{2}{|l|}{ Sex } \\
\hline Male & $87(51)$ \\
\hline Female & $85(49)$ \\
\hline \multicolumn{2}{|c|}{ Age (median 60) } \\
\hline$<60$ & $83(48)$ \\
\hline$\geqslant 60$ & $89(52)$ \\
\hline \multicolumn{2}{|l|}{ ECOG PS } \\
\hline 0 & $27(16)$ \\
\hline 1 & $137(80)$ \\
\hline 2 & $8(5)$ \\
\hline \multicolumn{2}{|l|}{ Metastases } \\
\hline 1 & $63(37)$ \\
\hline 2 & 55 (32) \\
\hline $3+$ & $54(31)$ \\
\hline \multicolumn{2}{|c|}{ Sites of metastases } \\
\hline Lung & $100(58)$ \\
\hline Liver & 65 (38) \\
\hline Bone & $48(28)$ \\
\hline Brain & $14(8)$ \\
\hline \multicolumn{2}{|c|}{ Baseline $\mathrm{LDH}\left(\mathrm{UI}^{-1}\right)$, reference range $\left(313-618 \mathrm{UI}^{-1}\right)$} \\
\hline$<618$ & $115(67)$ \\
\hline$\geqslant 618$ & $57(33)$ \\
\hline \multicolumn{2}{|c|}{ Baseline ANC $\left(\mathrm{K}_{\mu \mathrm{I}^{-1}}\right)$, reference range $\left(1.7-7.3 \mathrm{~K} \mu \mathrm{I}^{-1}\right)$} \\
\hline Normal (1.7-7.3) & $152(88)$ \\
\hline Elevated $(\geqslant 7.3)$ & $20(12)$ \\
\hline \multicolumn{2}{|c|}{ Baseline ALC $\left(\mathrm{K}_{\mu \mathrm{I}^{-1}}\right)$, reference range $\left(1.2-4.8 \mathrm{~K} \mu \mathrm{I}^{-1}\right)$} \\
\hline Normal (1.2-4.8) & $76(44)$ \\
\hline Low $(<1.2)$ & $96(56)$ \\
\hline \multicolumn{2}{|c|}{$\begin{array}{l}\text { Abbreviations: } \mathrm{ALC}=\text { absolute lymphocyte count; } \mathrm{ANC}=\text { absolute neutrophil count; } E C O G \\
\mathrm{PS}=\text { Eastern Cooperative Group performance status; } \mathrm{LDH}=\text { lactate dehydrogenase. Base- } \\
\text { line demographics of patients treated on phase } 1 \text { immune checkpoint inhibitor therapy. } \\
\text { Sex, age, ECOG PS, number of metastases, sites of metastases, baseline } L D H \text {, baseline } \\
\text { ANC, and baseline ALC are all listed. }\end{array}$} \\
\hline
\end{tabular}

\section{Table 2. Univariate results for all 17 prognostic factors} evaluated

\begin{tabular}{|c|c|c|}
\hline Parameter & $\mathrm{HR}(95 \% \mathrm{Cl})$ & $P$-value \\
\hline Age $>52$ years & $1.4(0.9-2.0)$ & 0.11 \\
\hline No. of metastases $>2$ & $1.4(1.0-2.0)$ & 0.069 \\
\hline Male gender & $0.8(0.6-1.2)$ & 0.30 \\
\hline Haemoglobin > 10.1 & $0.8(0.5,1.2)$ & 0.21 \\
\hline$A L C>1.8$ & $0.7(0.4,1.1)$ & 0.13 \\
\hline Lung metastases & $0.8(0.6,1.2)$ & 0.33 \\
\hline Bone metastases & $1.1(0.8,1.7)$ & 0.53 \\
\hline Brain metastases & $0.9(0.5,1.7)$ & 0.77 \\
\hline $\mathrm{ECOG}>1$ & $2.6(1.2-5.6)$ & 0.016 \\
\hline Albumin $>3.5$ & $0.5(0.3,0.8)$ & 0.0066 \\
\hline $\mathrm{LDH}>0.75 \times \mathrm{ULN}$ & $2.6(1.7,3.8)$ & $<0.0001$ \\
\hline$W B C>6.1$ & $1.8(1.3,2.6)$ & 0.0007 \\
\hline Platelet $>300$ & $1.9(1.3,2.8)$ & 0.0005 \\
\hline ANC $>4.9$ & $2.2(1.5,3.0)$ & $<0.0001$ \\
\hline$N L R>4$ & $2.1(1.4,2.9)$ & $<0.0001$ \\
\hline Liver metastases & $1.8(1.2,2.5)$ & 0.0013 \\
\hline $\mathrm{PLR}>498$ & $3.0(1.8,5.0)$ & $<0.0001$ \\
\hline \multicolumn{3}{|c|}{$\begin{array}{l}\text { Abbreviations: } A L C=\text { absolute lymphocyte count; } A N C=\text { absolute neutrophil count; } \mathrm{Cl}=\text { confi- } \\
\text { dence interval; } E C O G=\text { Eastern Cooperative } G r o u p ; H R=\text { hazard ratio; } L D H=\text { lactate dehydro- } \\
\text { genase; } N L R=\text { neutrophil/lymphocyte ratio; } P L R=\text { platelet/lymphocyte ratio; } U L N=\text { upper limit } \\
\text { of normal; } W B C=\text { white blood cells. Univariate analysis was performed on } 17 \text { clinical factors to } \\
\text { evaluate whether any factors were independently associated with overall survival. Hazard ratios } \\
\text { with } 95 \% \mathrm{Cls} \text { and } P \text { values are provided. } P<0.05 \text { was considered statistically significant. }\end{array}$} \\
\hline
\end{tabular}

Table 3. Multivariable Cox proportional hazards regression model used to develop MDACC ICI Prognostic Score

\begin{tabular}{|c|c|c|}
\hline Parameter & HR $(95 \% \mathrm{Cl})$ & $P$-value \\
\hline Age $>52$ years & $1.6(1.1,2.4)$ & 0.0290 \\
\hline$E C O G>1$ & $2.8(1.3,6.3)$ & 0.0110 \\
\hline $\mathrm{LDH}>0.75 \times \mathrm{ULN}$ & $2.1(1.4,3.2)$ & 0.0003 \\
\hline Platelet $>300$ & $1.8(1.2,2.8)$ & 0.0064 \\
\hline ANC $>4.9$ & $2.3(1.6,3.5)$ & 0.0005 \\
\hline $\mathrm{ALC}<1.8$ & $3.3(1.9,5.7)$ & 0.0004 \\
\hline Liver metastases & $1.8(1.2,2.6)$ & 0.0020 \\
\hline \multicolumn{3}{|c|}{$\begin{array}{l}\text { Abbreviations: } A L C=\text { absolute lymphocyte count; } A N C=\text { absolute neutrophil count; } \\
\mathrm{Cl}=\text { confidence interval; ECOG PS=Eastern Cooperative Group performance status; } \mathrm{HR}= \\
\text { hazard ratio; } \mathrm{LDH}=\text { lactate dehydrogenase; } \mathrm{MDACC} I \mathrm{Cl}=\mathrm{MD} \text { Anderson Cancer Center } \\
\text { Immune Checkpoint Inhibitor; ULN=upper limit of normal. Seven clinical variables } \\
\text { associated with significantly worse overall survival were incorporated into the MDA ICI } \\
\text { prognostic score. HRs are based on the retrospective analysis. }\end{array}$} \\
\hline
\end{tabular}

Table 4. Scoring patients with the MDA ICI Prognostic Score

Prognostic Scoring Index

\begin{tabular}{l|l} 
Prognostic factor & Points \\
\hline
\end{tabular}

Age

\begin{tabular}{r|r}
$<52$ & 0 \\
$>52$ & 1
\end{tabular}

\section{ECOG}

\begin{tabular}{l|l}
$<1$ & \\
$>1$ &
\end{tabular}

\section{LDH}

\begin{tabular}{|c|c}
\hline$<0.75 \times \mathrm{ULN}$ & 0 \\
$>0.75 \times \mathrm{ULN}$ & 1
\end{tabular}

\section{Platelet}

$<300$
$>300$

ANC

$<4.9$

$>4.9$

ALC

$<1.8$

$>1.8$

Liver metastases

No

Yes

Abbreviations: $A L C=$ absolute lymphocyte count; $A N C=$ absolute neutrophil count; ECOG = Eastern Cooperative Group; $L D H=$ lactate dehydrogenase; ULN = upper limit of normal. Table detailing how to score patients with the MD Anderson Immune Checkpoint Inhibitor prognostic score, whereby each clinical factor associated with negative survival is assigned one point.

$n=59$ ), and 4.3 months (MDACC score $3-5, n=35$ ), log-rank test $P<0.0001$ (Figure 2B). The c-index for predictability in our cohort was $0.61(0.04)$. Notably, the $95 \%$ confidence intervals of all three c-indexes all overlap.

\section{DISCUSSION}

Inclusion criteria for early phase trials universally mandate that patients have a life expectancy of at least 3 months. Prognostication of survival in clinic is quite challenging. Our analyses aimed to bring an easily calculable score to the clinic to aid in patient selection for ICI early phase trials. After a thorough analyses of 17 total clinical and laboratory parameters, we have developed a novel 
A

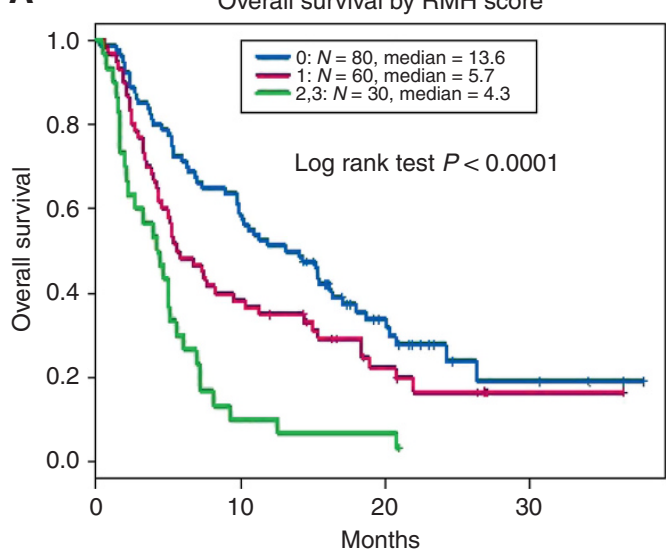

B

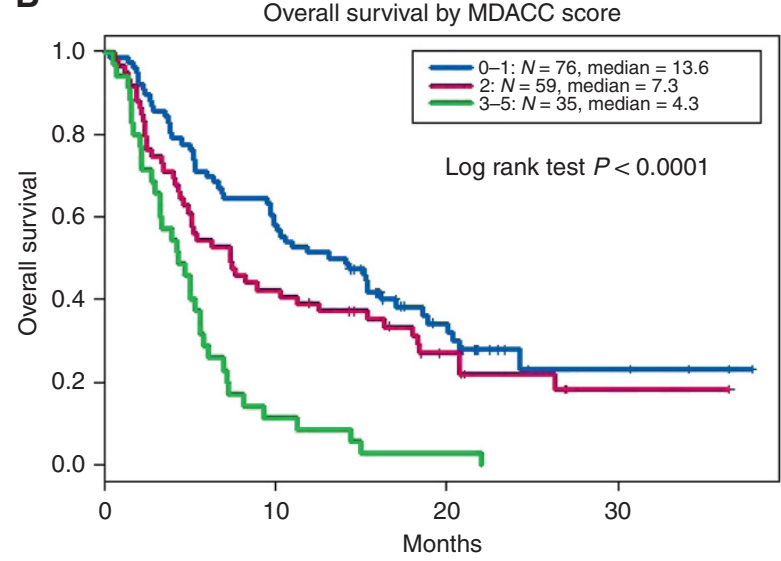

Figure 2. Overall survival by RMH and MDACC Score Kaplan-Meier survival analysis and Cox proportional hazard regression analysis for overall survival in patients treated on $\mathrm{ICl}$ phase 1 trials, by: (A) RMH risk score. The cohort is divided into three risk groups based on the number of factors present: 0 ( $n=80$, blue), 1 ( $n=60$, purple), $2-3(n=30$, green). (B) MDACC risk score. The cohort is divided into three risk groups based on the number of factors present: $0-1(n=76$, blue), 2 ( $n=59$, purple), $3-5$ ( $n=35$, green). ICl=immune checkpoint inhibitor therapy; MDACC=MD Anderson Cancer Center; RMH=Royal Marsden Hospital.

'MDA ICI' (MD Anderson Immune Checkpoint Inhibitor) prognostic scoring system with better predictability of OS than the RMH and MDACC scores. We incorporate seven clinical factors with prognostic significance for patients treated in phase 1 ICI trials and assign each factor one point: age $>52$ years, ECOG $>1, \mathrm{LDH}>0.75 \times \mathrm{ULN}$, platelet count $>300 \times 10^{3} \mu \mathrm{L}^{-1}$ ANC $>4.9 \times 10^{3} \mu \mathrm{L}^{-1}$, ALC, $<1.8 \times 10^{3} \mu \mathrm{L}^{-1}$ and liver metastases. Patients with the lowest risk score $(0-2)$ had significantly superior OS compared to patients with poorer risk scores $(>2)$. We report an OS of 7.3 months in patients treated on ICI-based phase 1 trials. This survival rate is consistent with previously reported phase 1 cohorts (Bachelot et al, 2000; Roberts et al, 2004; Arkenau et al, 2008). Similarly, our survival rates of patients at 6 and 12 months in this study were $56 \%$ and $38 \%$, which is comparable to historical rates of $43-70 \%$ at 6 months and $18-44 \%$ at 12 months (Bachelot et al, 2000; Wheler et al, 2012). The RMH prognostic scoring system identified elevated LDH levels, low albumin levels, and number of metastatic sites were independently associated with worse survival. The MDACC prognostic scoring system identified ECOG performance status and GI tumours to also be associated with worse survival. Although these prognostic scoring systems were developed and validated in the era of cytotoxic chemotherapy and targeted therapy, our results validate them in patients treated on checkpoint inhibitors, as well.

Interestingly, however, some of the negative prognostic factors identified in the RMH and MDA prognostic scoring systems, namely serum albumin levels and number of metastases, did not carry prognostic significance in patients treated with ICI-based therapy in multivariate analysis. In our analysis of albumin as a negative prognostic factor, a component of selection bias may be contributing to the discrepancy between our population and that treated at the RMH as the median albumin level in our cohort was $4.0 \mathrm{~g} \mathrm{dl}^{-1}$, which is higher than the median level of $3.3 \mathrm{~g} \mathrm{dl}^{-1}$ in the original RMH study (Arkenau et al, 2008). In our cohort, number of metastatic sites of disease also was not associated with poor prognosis. Like the Royal Marsden cohort, over 30\% of our patient population had widely metastatic cancers with three or more metastatic sites. Whether durability of response to checkpoint inhibitor therapy contributes to why number of metastatic sites of disease is not independently associated with survival remains to be seen. We also investigated whether certain sites of metastatic disease were associated with worse prognosis for patients enrolled in ICI phase 1 trials. Owing to concerns over blood brain barrier penetration and toxicity, patients with brain metastases are often excluded from most early phase immunotherapy trials and investigators often hesitate to place patients with any history of brain metastases on such trials. In our analysis, we identified no worse OS in patients with a history of brain metastases. As data on the effect of ICIs on patients with brain metastases begin to mature, it appears that the drugs may indeed have intracranial activity in at least melanoma and NSCLC, as well (Margolin et al, 2012). Early phase trials in other tumour types are currently ongoing. Interestingly, the presence of liver metastases was associated with worse OS. It has been postulated that the immune microenvironment of the liver, which must continually prevent undesirable immune responses to antigens absorbed in the gastrointestinal tract, is responsible for evading anti-tumour immunity (Revill, Testoni, Locarnini and Zoulim, 2016). For this reason, it is believed that in many tumour types, locoregional control of liver metastases yields reproducibly higher response rates than systemic therapy and is the largest determinant of survival in patients with liver metastases (Lemke et al, 2016). Whether combining checkpoint inhibitor therapy with systemic agents that modulate the liver microenvironment or liver directed therapies would improve outcomes in patients with liver metastases remains to be seen. Clinically, age is often used as an inclusion criteria in phase 1 trials. Interestingly, in our analysis age was picked up as a multivariate factor, but not a univariate factor. The fact that age was significant after adjustment for other study factors, but not on univariate analysis, highlights why univariate results are not considered in the construction of scoring systems. In this case, we believe that confounding was operating to mask the association between age and OS in univariate analysis.

Our data suggest that laboratory markers of inflammation are central to prognosticating patients enrolled on early phase ICI trials. Cancer-associated inflammation is considered to be a hallmark of cancer (Hanahan and Weinberg, 2011). LDH, a wellknown biomarker for inflammation (Agarwala et al, 2009) and cell death in the context of oxidative stress or hypoxic conditions(Le et al, 2010), was found to be prognostic in both the RMH and MDACC prognostic scoring systems. Not surprisingly, elevated $\mathrm{LDH}$ was found to be a negative prognostic marker in our analysis of patients treated with ICI-based therapy, as well. Interestingly, recursive partitioning analysis identified LDH $>75 \%$ of the upper limit of normal as the 'optimal' value with maximal difference in OS, suggesting that higher 'normal' values may be prognostic, as well. Although using an institutional upper limit of normal value for LDH may ultimately make application of the scoring system 
most user-friendly in a clinical setting, using 'optimal' cut-points generally produces a model with better predictive accuracy than simply using 'normal' values, and in this discovery set of patients even the highest $25 \%$ of 'normal' LDH values were associated with worse overall prognosis. Like LDH, neutrophilia, and lymphopenia have been identified as markers of cancer-related inflammation in the peripheral blood (Mantovani, Allavena, Sica and Balkwill, 2008). The quotient of the absolute neutrophil count and absolute lymphocyte count, the neutrophil/lymphocyte ratio (NLR), has been found to be independently prognostic for survival in advanced colorectal cancer (Walsh, Cook, Goulder, Justin and Keeling, 2005), gastric cancer (Yamanaka, Matsumoto, Teramukai, Ishiwata, Nagai and Fukushima, 2007), pancreatic cancer (An et al, 2010), prostate cancer, renal cell cancer (Keizman et al, 2012), and non-small cell lung cancer (Sarraf, Belcher, Raevsky, Nicholson, Goldstraw and Lim, 2009), as well. NLR has been found to hold negative prognostic value in patients treated on phase 1 trials (Kumar et al, 2015) and in melanoma patients treated with any form of immunotherapy (Zaragoza et al, 2016). Although the NLR did not demonstrate prognostic significance in our study, both neutrophilia and lymphopenia were associated with poor prognosis in multivariate analysis. Whether neutrophilia is a marker or mediator of response to ICI-based therapy remains to be explored. Elevated ANC in the peripheral blood of cancer patients has been associated with tumour-associated neutrophilia (Schmidt et al, 2005). Preclinical modulation of tumour-associated neutrophilia has previously demonstrated efficacy in tumour reduction (Pekarek, Starr, Toledano and Schreiber, 1995; Fridlender et al, 2009). ANC has also been hypothesised to be a good surrogate in peripheral blood for immunosuppressive myeloid-derived suppressor cells (MDSC), consisting of both neutrophils and immature myeloid cells (Brandau et al, 2011). By suppressing T-cell function, it is believed that tumours with higher population of MDSCs may not harbour the ideal immune microenvironment for ICI therapy to be effective. Similarly, thrombocytosis has been previously identified as an inflammation-based prognostic factor in multiple tumour types (Forrest, McMillan, McArdle, Angerson and Dunlop, 2003; Crumley, McMillan, McKernan, McDonald and Stuart, 2006). Although the reasons for this remain speculative at this time, data suggest that platelets may promote circulating tumour cells to evade the immune system via adhesion to the vascular endothelium (Heng et al, 2009).

Patient selection for early phase clinical trials remains challenging as patients typically have advanced disease and are refractory to multiple lines of therapy. However, survival of patients in a phase 1 clinic varies widely. Although inclusion criteria for early phase trials mandate that patients have a reasonable life expectancy, there are no models to objectively guide physicians in making this determination in patients who are being considered for ICI therapy phase 1 trials. Our analysis identified age, ECOG performance status, and the presence of liver metastases as negative clinical prognostic factors. Laboratory markers of inflammation, including elevated LDH, lymphopenia, neutrophilia, and thrombocytosis were identified as negative laboratory prognostic biomarkers.

We acknowledge that a single-institution, retrospective analysis has its limitations. Specifically, the variety of tumour types and ICI-based regimens utilised in phase 1 trials results in a heterogeneous population. In an attempt to minimise this heterogeneity, only anti-PD1 and anti-CTLA4 antibody-based regimens were included in this study. Owing to this restricted sample size, it should be noted that in this study there were only 7.4 deaths per variable analysed. For this reason, the individual variables should not be considered predictive when removed from the confines of this scoring system until prospectively validated in a larger cohort. To be clear, our novel scoring system is not meant to be a predictive biomarker, but indeed a prognostic tool to complement predictive biomarkers. Biomarkers predicting response to ICI-based therapy remains an unmet need. PD-1/ PD-L1 expression, tumour-infiltrating lymphocytes, MSI-status, and immune gene signatures are actively being investigated as predictive biomarkers of response to ICI therapy (Herbst et al, 2014; Chen et al, 2016). Predictive biomarkers such as these are used to estimate the probability for response to a given treatment and testing predictability of a test requires randomisation. We believe our novel prognostic scoring system compliments PD1/ PDL-1 expression, MSI status, and immune gene signatures from tumour biopsies in selecting patients for future ICI-based trials.

A prospective, multi-institution external validation of our scoring system is currently being planned as are further studies evaluating whether normalisation of ALC, ANC, LDH, and platelet count are associated with response to ICI-based therapy. Our study is intended to be generalisable across tumour types in a phase 1 clinic and included patients with 41 different tumour types. It is known that certain tumour types are inherently more aggressive and more responsive to immunotherapy than others. As more patients continue to be enrolled on ICI-based phase 1 trials, we anticipate that tumour specific analyses will be feasible in the near future, as well.

\section{ACKNOWLEDGEMENTS}

The authors acknowledge the patients and their families for participating in this study. This work was supported in part by The Cancer Prevention and Research Institute of Texas (RP1100584), the Sheikh Khalifa Bin Zayed Al Nahyan Institute for Personalized Cancer Therapy, 1U01 CA180964, NCATS Grant UL1 TR000371 (Center for Clinical and Translational Sciences), The Bosarge Family Foundation, 'The Beck family', Robert Beck and Merry Beck and the MD Anderson Cancer Center Support Grant (P30 CA016672). The funders had no role in the design of the study; the collection, analysis, and interpretation of the data; the writing of the manuscript; and the decision to submit the manuscript for publication.

\section{CONFLICT OF INTEREST}

Vivek Subbiah receives research funding for clinical trials from Roche/Genentech, Novartis, Bayer, GSK, Nanocarrier, Vegenics, Northwest Biotherapeutics, Berghealth, Incyte, Fujifilm, Pharmamar, D3, Pfizer, Multivir, Amgen, Abbvie, Bluprint medicines. This was presented in part at the 2017 American Association for Cancer Research (AACR) Annual Meeting.

\section{REFERENCES}

Agarwala SS, Keilholz U, Gilles E, Bedikian AY, Wu J, Kay R et al. (2009) $\mathrm{LDH}$ correlation with survival in advanced melanoma from two large, randomised trials (Oblimersen GM301 and EORTC 18951). Eur J Cancer 45: $1807-1814$.

An X, Ding PR, Li YH, Wang FH, Shi YX, Wang ZQ et al. (2010) Elevated neutrophil-to-lymphocyte ratio predicts survival in advanced pancreatic cancer. Biomarkers 15: 516-522.

Arkenau HT, Olmos D, Ang JE, de Bono J, Judson I, Kaye S (2008) Clinical outcome and prognostic factors for patients treated within the context of a phase I study: the Royal Marsden Hospital experience. $\mathrm{Br}$ J Cancer 98: 1029-1033.

Bachelot T, Ray-Coquard I, Catimel G, Ardiet C, Guastalla JP, Dumortier A et al. (2000) Multivariable analysis of prognostic factors for toxicity and survival for patients enrolled in phase I clinical trials. Ann Oncol 11: $151-156$. 
Borghaei H, Paz-Ares L, Horn L, Spigel DR, Steins M, Ready NE et al. (2015) Nivolumab versus docetaxel in advanced nonsquamous non-small-cell lung cancer. N Engl J Med 373: 1627-1639.

Brandau S, Trellakis S, Bruderek K, Schmaltz D, Steller G, Elian M et al. (2011) Myeloid-derived suppressor cells in the peripheral blood of cancer patients contain a subset of immature neutrophils with impaired migratory properties. J Leukoc Biol 89: 311-317.

Chen PL, Roh W, Reuben A, Cooper ZA, Spencer CN, Prieto PA et al. (2016) Analysis of immune signatures in longitudinal tumor samples yields insight into biomarkers of response and mechanisms of resistance to immune checkpoint blockade. Cancer Discov 6: 827-837.

Crumley AB, McMillan DC, McKernan M, McDonald AC, Stuart RC (2006) Evaluation of an inflammation-based prognostic score in patients with inoperable gastro-oesophageal cancer. Br J Cancer 94: 637-641.

Forrest LM, McMillan DC, McArdle CS, Angerson WJ, Dunlop DJ (2003) Evaluation of cumulative prognostic scores based on the systemic inflammatory response in patients with inoperable non-small-cell lung cancer. Br J Cancer 89: 1028-1030.

Fridlender ZG, Sun J, Kim S, Kapoor V, Cheng G, Ling L et al. (2009) Polarization of tumor-associated neutrophil phenotype by TGF-beta: 'N1' versus 'N2' TAN. Cancer Cell 16: 183-194.

Hanahan D, Weinberg RA (2011) Hallmarks of cancer: the next generation. Cell 144: 646-674.

Harrell Jr. FE, Lee KL, Mark DB (1996) Multivariable prognostic models: issues in developing models, evaluating assumptions and adequacy, and measuring and reducing errors. Stat Med 15: 361-387.

Heng DY, Xie W, Regan MM, Warren MA, Golshayan AR, Sahi C et al. (2009) Prognostic factors for overall survival in patients with metastatic renal cell carcinoma treated with vascular endothelial growth factortargeted agents: results from a large, multicenter study. J Clin Oncol 27: 5794-5799.

Herbst RS, Soria JC, Kowanetz M, Fine GD, Hamid O, Gordon MS et al. (2014) Predictive correlates of response to the anti-PD-L1 antibody MPDL3280A in cancer patients. Nature 515: 563-567.

Keizman D, Gottfried M, Ish-Shalom M, Maimon N, Peer A, Neumann A et al. (2012) Pretreatment neutrophil-to-lymphocyte ratio in metastatic castration-resistant prostate cancer patients treated with ketoconazole: association with outcome and predictive nomogram. Oncologist 17: 1508-1514

Kumar R, Geuna E, Michalarea V, Guardascione M, Naumann U, Lorente D et al. (2015) The neutrophil-lymphocyte ratio and its utilisation for the management of cancer patients in early clinical trials. $\mathrm{Br} J$ Cancer 112: $1157-1165$.

Larkin J, Chiarion-Sileni V, Gonzalez R, Grob JJ, Cowey CL, Lao CD et al. (2015) Combined nivolumab and ipilimumab or monotherapy in untreated melanoma. $N$ Engl J Med 373: 23-34.

Le A, Cooper CR, Gouw AM, Dinavahi R, Maitra A, Deck LM et al. (2010) Inhibition of lactate dehydrogenase A induces oxidative stress and inhibits tumor progression. Proc Natl Acad Sci USA 107: 2037-2042.
Lemke J, Cammerer G, Ganser J, Scheele J, Xu P, Sander S et al. (2016) Survival and prognostic factors of colorectal liver metastases after surgical and nonsurgical treatment. Clin Colorectal Cancer 15: e183-e192.

Mantovani A, Allavena P, Sica A, Balkwill F (2008) Cancer-related inflammation. Nature 454: 436-444.

Margolin K, Ernstoff MS, Hamid O, Lawrence D, McDermott D, Puzanov I et al. (2012) Ipilimumab in patients with melanoma and brain metastases: an open-label, phase 2 trial. Lancet Oncol 13: 459-465.

Marshall RJ (2001) The use of classification and regression trees in clinical epidemiology. J Clin Epidemiol 54: 603-609.

Michot JM, Bigenwald C, Champiat S, Collins M, Carbonnel F, Postel-Vinay S et al. (2016) Immune-related adverse events with immune checkpoint blockade: a comprehensive review. Eur J Cancer 54: 139-148.

Pekarek LA, Starr BA, Toledano AY, Schreiber H (1995) Inhibition of tumor growth by elimination of granulocytes. J Exp Med 181: 435-440.

Revill P, Testoni B, Locarnini S, Zoulim F (2016) Global strategies are required to cure and eliminate HBV infection. Nat Rev Gastroenterol Hepatol 13: 239-248.

Roberts Jr. TG, Goulart BH, Squitieri L, Stallings SC, Halpern EF, Chabner BA et al. (2004) Trends in the risks and benefits to patients with cancer participating in phase 1 clinical trials. JAMA 292: 2130-2140.

Sarraf KM, Belcher E, Raevsky E, Nicholson AG, Goldstraw P, Lim E (2009) Neutrophil/lymphocyte ratio and its association with survival after complete resection in non-small cell lung cancer. J Thorac Cardiovasc Surg 137: 425-428.

Schmidt H, Bastholt L, Geertsen P, Christensen IJ, Larsen S, Gehl J et al. (2005) Elevated neutrophil and monocyte counts in peripheral blood are associated with poor survival in patients with metastatic melanoma: a prognostic model. Br J Cancer 93: 273-278.

Walsh SR, Cook EJ, Goulder F, Justin TA, Keeling NJ (2005) Neutrophillymphocyte ratio as a prognostic factor in colorectal cancer. J Surg Oncol 91: 181-184.

Wheler J, Tsimberidou AM, Hong D, Naing A, Falchook G, Piha-Paul S et al. (2012) Survival of 1,181 patients in a phase I clinic: the MD Anderson Clinical Center for targeted therapy experience. Clin Cancer Res 18: 2922-2929.

Yamanaka T, Matsumoto S, Teramukai S, Ishiwata R, Nagai Y, Fukushima M (2007) The baseline ratio of neutrophils to lymphocytes is associated with patient prognosis in advanced gastric cancer. Oncology 73: 215-220.

Zaragoza J, Caille A, Beneton N, Bens G, Christiann F, Maillard H et al. (2016) High neutrophil-to-lymphocyte ratio measured before starting ipilimumab treatment is associated with reduced overall survival in patients with melanoma. Br J Dermatol 174: 146-151.

This work is published under the standard license to publish agreement. After 12 months the work will become freely available and the license terms will switch to a Creative Commons AttributionNonCommercial-Share Alike 4.0 Unported License.

Supplementary Information accompanies this paper on British Journal of Cancer website (http://www.nature.com/bjc) 